\title{
Successful Treatment of a Case of Unilateral Endogenous Klebsiella pneumoniae Endophthalmitis
}

\author{
Omer Takes, ${ }^{1}$ Gamze Kocaoglu, ${ }^{1}$ Ziya Ayhan ${ }^{2}$ and A Osman Saatci ${ }^{3}$ \\ 1. Resident; 2. Fellow; 3. Professor, Department of Ophthalmology, Dokuz Eylul University, Izmir, Turkey
}

\begin{abstract}
A 52-year-old woman with diabetes was examined for acute visual loss and ocular pain in oculus dexter (OD). Two weeks earlier, a nephrostomy catheter was inserted for the treatment of nephrolithiasis and 10 days after this procedure she suffered an acute myocardial infarction. During hospitalisation, she developed a high fever and Klebsiella pneumoniae was detected in the blood cultures. On eye examination, mild corneal oedema, minimal hypopyon, vitritis and subretinal abscess of 3DD size at the uppertemporal retina were noted in the right eye. Single intravitreal injection of vancomycin, ceftazidime and clindamycin was given in addition to systemic antibiotic treatment for 14 days. Intraocular inflammation resolved rapidly within days and she regained a visual acuity of 8/10 with a residual chorioretinal scar 2 months later. Endogenous endophthalmitis can be treated successfuly if the diagnosis can be established early enough and treated accordingly.
\end{abstract}

\section{Keywords}

Endogenous endophthalmitis, intravitreal injection, Klebsiella pneumoniae, retina, uveitis

\begin{abstract}
Disclosure: Omer Takes, Gamze Kocaoglu, Ziya Ayhan and A Osman Saatci have no conflicts of interest to declare. No funding was received in the publication of this article. open Access: This article is published under the Creative Commons Attribution Noncommercial License, which permits any non-commercial use, distribution, adaptation and reproduction provided the original author(s) and source are given appropriate credit.

Compliance with Ethics Guidelines: Informed consent was received from the patient involved in the case study.

Received: 11 June 2015 Accepted: 3 July 2015 Citation: European Ophthalmic Review, 2015;9(1):23-4 DOl: 10.17925/EOR.2015.09.01.23

Correspondence: A Osman Saatci, Mustafa Kemal Pasa Bulvari, No. 73, A blok, Daire: 9, Narlidere, 35320, Izmir, Turkey. E: osman.saatci@yahoo.com
\end{abstract}

Endogenous endophthalmitis is a rare inflammatory condition of the intraocular cavity that is caused by the haematogenous spread of bacterial and/or fungal agents from the distant infectious loci mostly located in liver, lung, endocardium, meninges and urinary tract. ${ }^{1,2}$ It is commonly associated with an underlying immunosuppressive status including diabetes mellitus, cardiac disease, renal insufficiency and malignancy. Gram-negative bacteriae are relatively more frequent than Gram-positive bacteriae, particularly in Asia. ${ }^{3}$

We report a successfully treated case of 52-year-old woman with diabetes who developed Klebsiella pneumoniae septicaemia secondary to nephrostomy and subsequent unilateral endogenous endophthalmitis.

\section{Case Report}

A 52-year-old woman with 20 years history of poorly controlled diabetes experienced acute visual loss and ocular pain in her right eye. Two weeks earlier a nephrostomy catheter was inserted for the treatment of nephrolithiasis. Ten days after this procedure she suffered an acute myocardial infarction and was hospitalised. Three days after the hospitalisation she developed the above-mentioned ocular symptoms in her right eye in association with high fever. She also had herpes labialis for 2 days. On eye examination, best-corrected visual acuity was 1/10 in oculus dexter (OD) and 20/20 in oculus sinister (OS). Slit-lamp examination showed mild corneal oedema, +++ cells, minimal hypopyon and mild to moderate vitritis in OD. The anterior segment was unremarkable in OS. Intraocular pressure was within normal limit oculus uterque (OU). Fundoscopy revealed a subretinal abscess of almost 3DD at the uppertemporal retina in OD and a few cotton-wool spots at the posterior pole in OS (see Figure 1). Blood cultures already demonstrated K. pneumoniae. Our diagnosis was unilateral K. pneumoniae endophthalmitis (KPEE). She was treated with a single intravitreal injection of vancomycin + ceftazidime and clindamycin in association with the systemic antibiotics (gentamicin + daptomycin and acyclovir) for 14 days. Vitreous culture turned out to be negative. Topical cyclopentolate and prednisolone acetate drops were also administered.

Intraocular inflammation resolved rapidly within days and she regained visual acuity of $8 / 10$ with a residual choroiretinal scar 2 months later (see Figure 2).

\section{Discussion}

Among inpatients with haematogenous infection, the overall incidence of presumed endogenous endophthalmitis was $0.04 \%$ among patients with bacteraemia. ${ }^{4}$ In a recent review of 342 cases of endogenous bacterial endophthalmitis reported between 1986 and 2012 predisposing conditions were present in $60 \%$ of cases. ${ }^{2}$ The median final visual acuity was 20/100 with $44 \%$ of eyes achieving visual acuity worse than 20/200. Among all cases, $24 \%$ required evisceration or enucleation and the mortality was $4 \%$. The most common causative Gram-negative bacteria were $K$. pneumonia, Pseudomonas aeruginosa and Neisseria meningitidis and the most common causative Grampositive bacteria were Staphylococcus aureus, group B Streptococcus, Streptococcus pneumoniae and Nocardia species, respectively. 


\section{Figure 1: Clinical Appearance at Presentation}

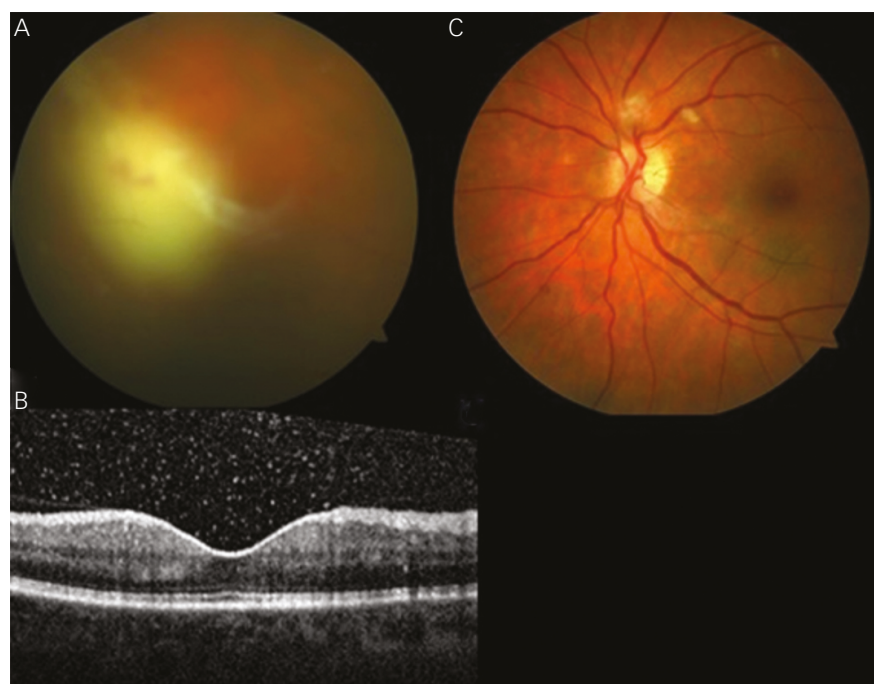

A. Right eye: colour fundus picture showing the subretinal abscess of almost 3DD at the uppertemporal retina. B. Optical coherence tomography of the right eye showing normal foveal contour and multiple hyperreflective dots in the posterior vitreous corresponding to the inflammatory cells. C. Left eye: colour fundus picture showing the cotton-wool spots at the posterior pole.

Visual outcome following endogenous bacterial endophthalmitis is usually poor. A study of 22 consecutive patients by Yang et al. ${ }^{5}$ showed that $89 \%$ of patients achieved a visual acuity of light perception at best, with $41 \%$ requiring enucleation or evisceration. Previously, reported anectodal cases with KPEE were generally with a poor visual outcome. ${ }^{6-10}$ Occasionally, relatively good visual outcome was reported in cases with KPEE. ${ }^{11,12}$ In a retrospective review of 289 patients with clinical diagnosis of pyogenic liver abscess, 10 patients (3.5\%) with the complication of KPEE were discovered. ${ }^{13}$ Among them, five patients (50 \%) had no light perception, one (light perception) and four (hand movement vision). Ang et al. ${ }^{14}$ reviewed all consecutive patients with KPEE over 20 years. Their study population comprised 71 eyes of 61 patients. While $80 \%$ had vision worse than 4/200, $26.8 \%$ required evisceration. The authors concluded that patients presenting with hypopyon and unilateral involvement had a poorer visual prognosis. Yoon et al. ${ }^{15}$ reviewed seven cases of KPEE.
Figure 2: Resolution of the Subretinal Abscess During the Follow-up

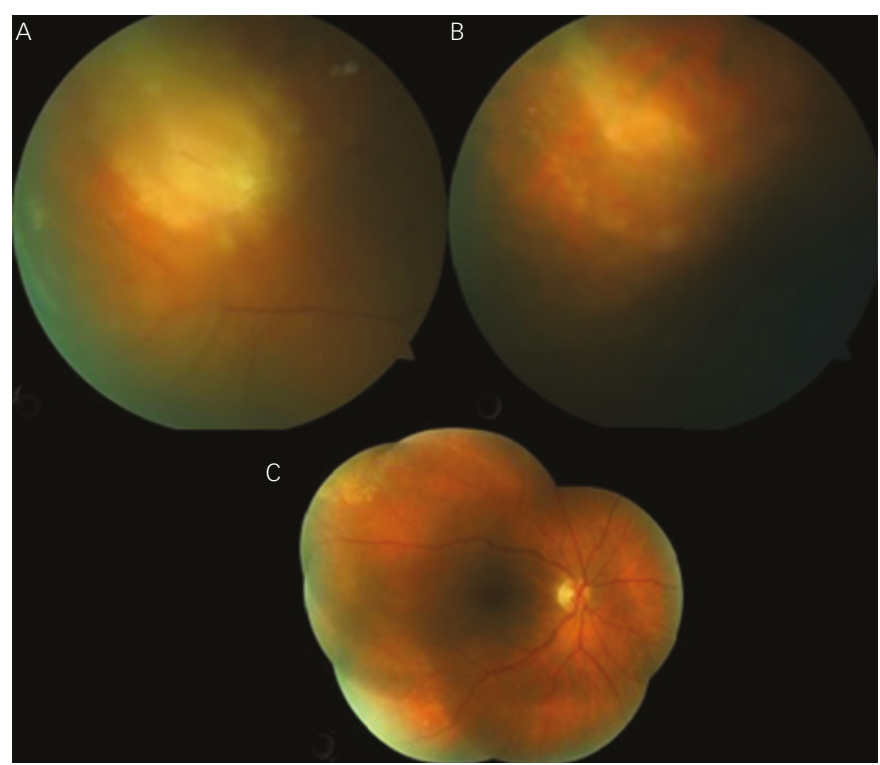

A. Right eye: colour fundus picture at the third day after the intravitreal antibiotic injection. B. Colour fundus picture at the fifth day after the intravitreal antibiotic injection. C. Composite colour picture of the right eye 2 months after the intravitreal antibiotic injection.

Five patients (71\%) had diabetes and four (57\%) liver abscess. Eighty per cent of eyes had hand movements or worse vision at the presentation. After aggressive therapy including pars plana vitrectomy $70 \%$ of eyes had counting fingers vision or worse.

Early recognition of endogenous endophthalmitis is crucial. Therefore clinicians should consider this diagnosis in patients presenting with the suggestive ocular symptoms and having the risk factors for endogenous endophthalmitis. Prompt investigation and meticulous treatment should be instituted as soon as the suspicion arises. Our case clearly is a good clinical example showing the importance of how the proper approach can affect the visual outcome positively in eyes with KPEE.
1. Jackson TL, Eykyn SJ, Graham EM, Stanford MR, Endogenous bacterial endophthalmitis: a 17-year prospective series and review of 267 reported cases, Surv Ophthalmol, 2003;48:403-23.

2. Jackson TL, Paraskevopoulos T, Georgalas I, Systematic review of 342 cases of endogenous bacterial endophthalmitis, Surv Ophthalmol, 2014:59:627-35.

3. Kashani AK, Eliott D, The emergence of Klebsiella pneumoniae endogenous endophthalmitis in the USA: basic and clinical advances, J Ophthalmic Inflamm Infect, 2013;3:28.

4. Vaziri K, Pershing S, Albini TA, et al., Risk factors predictive of endogenous endophthalmitis among hospitalized patients with hematogenous infections in the United States, Am J ophtalmol, 2015:159:498-504

5ang CS, Tsai HY, Sung CS, et al. Endogenous Klebsiella endophthalmitis associated with pyogenic liver abscess,

\section{Ophthalmology, 2007:114:876-80}

Sharma M, Chow DR, Muller MP, Endogenous Klebsiella endophthalmitis in a Vietnamese immigrant, CMAJ, 2009:181:495-7.

7. Al-Amri MS, Endogenous endophthalmitis secondary to pyogenic liver abscess, Int J Diabetes Mellit, 2010:2:64-6.

8. Dehghani AR, Masjedi A, Fazel F, et al., Endogenous Klebsiella endophthalmitis associated with liver abscess: first case report from Iran, Case Rep Ophthalmol, 2011:2:10-4.

9. Sheu SJ, Kung YH, Wu TT, et al., Risk factors for endogenous endophthalmitis secondary to Klebsiella pneumoniae liver abscess: 20-year experience in Southern Taiwan, Retina, 2011:31:2026-31

10. Soon WC, Pouncey A, Ashley E, Bowen EF, Klebsiella pneumoniae infection: A virulent cause of visual loss, Case
Rep Ophthalmol 2014:5:468-73.

11. Ishii K, Hiraoka T, Kaji Y, et al., Successful treatment of endogenous Klebsiella pneumoniae endophthalmitis: a case report, Int Ophthalmol, 2011;31:29-31.

12. Siu GD, LO EC, Young A, Endogenous endophthalmitis with visual acuity of 6/6, BMJ Case Rep, 2015 Mar 18;2015.

13. Tan YM, Chee SP, Soo KC, Chow P, Ocular manifestations and complications of pyogenic liver abscess, World I Surg. 2004;28:38-42.

14. Ang M, Jap A, Chee SP, Prognostic factors and outcomes in endogenous Klebsiella pneumoniae endophthalmitis, Am Ophthalmol, 2011;151:338-44.

15. Yoon YH, Lee SU, Sohn JH, Lee SE, Result of early vitrectomy for endogenous Klebsiella pneumoniae endophthalmitis, Retina, 2003;23:366-70. 